\title{
VERIFICAC̣ÃO DA ATIVIDADE CONDILAR EM PACIENTES COM PADRÃO ESQUELÉTICO CLASSE III POR INTERMÉDIO DA CINTILOGRAFIA ÓSSEA*
}

\author{
Larissa Perales Bittencourt ${ }^{1}$, Sérgio Augusto Lopes Souza², Mônica Magnanini ${ }^{3}$, \\ Léa Mirian Barbosa da Fonseca ${ }^{4}$, Bianca Gutfilen ${ }^{5}$
}

Resumo OBJETIVO: Utilizar a cintilografia óssea facial para identificar o crescimento esquelético mandibular, através do metabolismo ósseo condilar de indivíduos com crescimento craniofacial equilibrado, e compará-lo à atividade condilar daqueles com crescimento mandibular excessivo. MATERIAIS E MÉTODOS: Quarenta e sete indivíduos, de ambos os sexos, entre 18 e 28 anos de idade, foram divididos em grupo controle classe I $(n=13)$ - e grupo caso - classe III ( $n=34)$. As imagens foram obtidas duas horas após injeção intravenosa de $200 \mu \mathrm{Ci} / \mathrm{kg}$ de ${ }^{99 \mathrm{~m}} \mathrm{Tc}$-MDP. Foram realizadas incidências laterais do crânio e posterior da coluna lombar e a taxa de contagem foi determinada pela obtenção da média de contagem dos côndilos e da quarta vértebra lombar utilizando 300.000 contagens. RESULTADOS: Não foram encontradas captações condilares assimétricas em ambos os grupos, e apesar da ausência de significância estatística, os valores médios de captação condilar foram maiores no grupo caso. CONCLUSÃO: A média de captação aumentada indicou maior metabolismo ósseo condilar nos indivíduos classe III, sugerindo um provável crescimento mandibular residual. Mais estudos estão sendo realizados para aumentar esta amostragem.

Unitermos: Cintilografia óssea; Côndilo mandibular; Crescimento. Articulação temporomandibular.

\begin{abstract}
Skeletal scintigraphy for assessment of condylar uptake in class III malocclusion.
OBJECTIVE: To use skeletal scintigraphy to detect mandibular growth through the analysis of bone metabolism in individuals with normal craniofacial growth, and to compare the findings with those seen in individuals with excessive craniofacial growth. MATERIALS AND METHODS: Forty-seven male and female patients between 18 to 28 years of age were divided in two groups - class I, control ( $n=13$ ), and class III, case $(\mathrm{n}=34)$. Images were obtained two hours after intravenous injection of ${ }^{99 \mathrm{~m}} \mathrm{Tc}-\mathrm{MDP}(200 \mu \mathrm{Ci} / \mathrm{kg})$. Right and left side views of the mandible were obtained as well as a posterior view of the lumbar spine. The count rate was determined by the mean values of the count of the condyles and of the fourth lumbar vertebra, using a total of 300,000 counts. RESULTS: No asymmetrical condyle uptake was observed in both groups, and in spite of the absence of statistically significant differences, the mean condylar uptake values were higher in class III group. CONCLUSION: The increased mean uptake indicated higher condylar bone metabolism in class III individuals suggesting residual mandibular growth. Further studies are presently ongoing to increase our sample size.
\end{abstract}

Key words: Bone scintigraphy; Mandibular condyle; Mandibular growth; Temporomandibular joint.

\section{INTRODUÇÃO}

O crescimento facial tem sido, ao longo dos anos, objeto de estudo sob diversos métodos. Um dos mais utilizados é a aná-

* Trabalho realizado no Departamento de Radiologia da Faculdade de Medicina da Universidade Federal do Rio de Janeiro (FM-UFRJ), Rio de Janeiro, RJ.

1. Doutoranda em Radiologia da FM-UFRJ, Aluna de Especialização do Curso de Ortodontia da Universidade Gama Filho (UGF).

2. Doutorando em Radiologia da FM-UFRJ.

3. Estatística do Núcleo de Estudo em Saúde Coletiva (NESC) da UFRJ.

4. Professora Titular de Medicina Nuclear da FM-UFRJ.

5. Professora Adjunta do Departamento de Radiologia da FMUFR.

Endereço para correspondência: Dra. Larissa Perales Bittencourt. Rua Assis Brasil, 143, BI. 02, ap. 201, Copacabana. Rio de Janeiro, RJ, 22230-010. E-mail: larissab@olimpo.com.br

Recebido para publicação em 1/9/2004. Aceito, após revisão, em 9/12/2004. lise cefalométrica seriada, que se faz a partir da sobreposição de traçados cefalométricos. Embora valioso, este método indica apenas o crescimento passado e requer um acompanhamento longitudinal $^{(\mathbf{1 - 3})}$. A radiografia de mão e punho é outro procedimento bastante aplicado na determinação do status da maturação esquelética corporal. A desvantagem aqui encontrada é a necessidade de extrapolar as informações para o crescimento facial ${ }^{(3-6)}$.

A predição do crescimento facial é de importância vital nos casos de deformidades esqueléticas dentofaciais ou craniofaciais $^{(\mathbf{1}, \mathbf{7 - 9})}$, como também nos casos de assimetria causada por hiper ou hipoplasia condilar $^{(\mathbf{1 , 2})}$. Com esse conhecimento, o momento, o tipo e o porte da cirurgia po- dem ser melhor definidos, ou seja, se a cirurgia deverá ser realizada em idade precoce e de forma menos invasiva para os casos mais graves, ou se a finalização do crescimento será aguardada e a intervenção será corretiva, reduzindo as chances de recidiva e de um segundo tempo operatório $^{(\mathbf{1}, 10)}$. Para o ortodontista, conhecer o potencial de crescimento do seu paciente cirúrgico pode influenciar na mecânica ortodôntica adotada, na finalização do caso e na orientação ao paciente quanto às suas limitações e expectativas.

$\mathrm{O}$ conhecimento dos mecanismos e períodos ativos do crescimento craniofacial é de grande importância para os ortodontistas, uma vez que o tratamento de muitas más-oclusões, denominadas esqueléticas, 
dependem do início do crescimento ativo ou do término deste. Durante muitos anos, a cartilagem condilar foi considerada um centro de crescimento primário, atuando de modo similar à lâmina epifisária dos ossos longos. Diferenças histológicas e biológicas, entretanto, descartaram esta teoria. Alguns autores postulam que a matriz condilar permita uma maior suscetibilidade às forças ambientais ${ }^{(11)}$.

A má-oclusão de classe III é um problema esquelético que reflete o desequilíbrio ântero-posterior das bases ósseas (maxila e mandíbula) em relação à base do crânio $^{(\mathbf{8 , 9})}$. O sulco mesiovestibular do primeiro molar permanente inferior encontra-se mesializado em relação à cúspide mesiovestibular do primeiro molar superior ${ }^{(\mathbf{1 2})}$. A má-oclusão, porém, não está restrita às arcadas, envolvendo o complexo craniofacial e acarretando desarmonia facial ${ }^{(\mathbf{1 3})}$.

Diferentemente de outras desarmonias esqueléticas que sofrem grande influência do ambiente, a má-oclusão de classe III possui grande tendência familiar. Os fatores herdados não somente influem no tamanho da mandíbula, mas também no tamanho da maxila, no comprimento e angulação da base craniana e na posição da cavidade glenóide. Entretanto, a extensão da influência do meio ambiente no crescimento mandibular ainda não é totalmente conhecida $^{(14)}$.

O prognatismo mandibular é normalmente corrigido através da combinação de tratamento ortodôntico e cirúrgico. A cirurgia mandibular precoce tem pouco ou nenhum efeito inibitório sobre o crescimento futuro. Por esta razão, a cirurgia ortognática deve ser retardada até que o crescimento tenha sido completado, essencialmente naqueles com problemas de crescimento excessivo. Caso contrário, pode ser esperada a recidiva da correção e a necessidade de retratamento ${ }^{(15-17)}$.

A cintilografia óssea surge no contexto odontológico como um método de diagnóstico que proporciona às diversas especialidades médicas a possibilidade de detecção de doenças ou de alterações metabólicas. A cintilografia pode documentar tanto o metabolismo normal como as alterações fisiológicas. Pode ainda ser efetiva como método de verificação do crescimento facial $^{(2,10)}$, na aplicabilidade de apare- lhos ortopédicos ${ }^{(\mathbf{1 8})}$, no acompanhamento da ósseo-integração de implantes dentários e no diagnóstico e localização de doenças infecciosas como a osteomielite ${ }^{(\mathbf{1 9})}$. Uma das mais importantes vantagens desse exame está na habilidade de obter informações fisiológicas do crescimento dinâmico com apenas uma observação ${ }^{(2)}$.

A cintilografia óssea tem várias aplicações na odontologia, como na avaliação da ósseo-integração de implantes metáli$\cos ^{(19)}$, ocorrendo maior concentração do radiofármaco nas regiões implantadas até o momento da ósseo-integração, na verificação da eficácia de tratamentos ortodônticos, através do uso de aparelhos que estimulem a atividade osteogênica, como, por exemplo, o aparelho de Herbst ${ }^{(\mathbf{1 8})}$, ou ainda na definição do tratamento da hiperplasia condilar ${ }^{(\mathbf{2 0})}$. Neste último caso, a escolha da técnica cirúrgica poderá depender da atividade condilar ${ }^{(10)}$.

No Brasil, o uso da cintilografia óssea para avaliação da atividade condilar é pouco conhecido. Por este motivo, e principalmente por se tratar de uma técnica simples, eficaz na detecção de qualquer alteração do metabolismo ósseo e de baixo custo, desenvolvemos uma metodologia para verificar a atividade condilar de pacientes com prognatismo mandibular, baseada na experiência de Kaban et al. ${ }^{(2)}$.

Este trabalho teve como objetivo definir as médias de captação condilar de indivíduos com padrão esquelético de classe I e classe III, além de realizar comparação entre ambos os grupos.

\section{MATERIAIS E MÉTODOS}

Esta pesquisa baseou-se em estudo do tipo transversal, com verificação do crescimento esquelético facial, através do metabolismo ósseo condilar em indivíduos sob tratamento ortodôntico, oriundos do Curso de Pós-Graduação da Universidade Gama Filho (UGF) e do Serviço de Cirurgia Bucomaxilofacial do Hospital Universitário Pedro Ernesto (HUPE) da Universidade do Estado do Rio de Janeiro (UERJ). A cintilografia óssea foi realizada no Serviço de Medicina Nuclear do Hospital Universitário Clementino Fraga Filho (HUCFF) da Universidade Federal do Rio de Janeiro (UFRJ).
O projeto foi submetido e aprovado pelo Comitê de Ética em Pesquisa do HUCFF-UFRJ.

A amostra constou de 47 indivíduos, de ambos os sexos, com idade entre 18 e 28 anos, separados em grupo controle - com padrão esquelético do tipo classe I $(n=13)$ - e grupo caso - com padrão esquelético de classe III $(n=34)$. O padrão esquelético foi determinado utilizando a análise cefalométrica de Steiner ${ }^{(21)}$.

Para a seleção da amostra foram incluídos indivíduos com radiografia de mão e punho revelando maturidade esquelética; idade cronológica maior que 18 anos; análise cefalométrica com $\mathrm{ANB}=2^{\circ}\left( \pm 2^{\circ}\right)$ grupo controle - e análise cefalométrica com $\mathrm{ANB}>4^{\circ}, \mathrm{SNB}>82^{\circ}, \mathrm{SND}>76 / 7^{\circ}$ - grupo caso.

Foram excluídos os pacientes com síndromes ou más-formações craniomaxilofaciais, neoplasias, doenças ósseas metabólicas, problemas endócrinos e desordens temporomandibulares.

Foi realizada administração intravenosa de $200 \mu \mathrm{Ci} / \mathrm{kg}$ de ${ }^{99 \mathrm{~m}} \mathrm{Tc}-\mathrm{MDP}$. As imagens foram obtidas em gama-câmera da marca Siemens (Diacam, colimador de alta resolução e baixa energia - Alemanha) duas horas após a injeção. Os pacientes tiveram a cabeça posicionada em hiperextensão, para evitar superposição do côndilo mandibular com a coluna cervical. A região de côndilo, ramo e corpo da mandíbula estiveram sob o campo de visão do detector da gama-câmera.

A técnica utilizada foi baseada e adaptada da técnica descrita por Kaban et al. ${ }^{\text {(2) }}$ em 1982. Foram realizadas incidências estáticas (planares) laterais (direita e esquerda) da cabeça, com a boca fechada e posterior da coluna lombar. As imagens foram obtidas com 300.000 contagens cada, método descrito na literatura ${ }^{(2)}$. Foi utilizada matriz no formato de $256 \times 256$. O exame foi realizado sempre pelo mesmo operador, de acordo com protocolo de exame.

As áreas de interesse (ROI) foram desenhadas sobre a imagem dos côndilos bilateralmente, sobre a quarta vértebra lombar e outra lateral à mesma (Figura 1). Esta última foi efetuada para quantificar o padrão de radiação de fundo (BKG). A taxa de contagem foi determinada em todas as regiões selecionadas, através da média de 


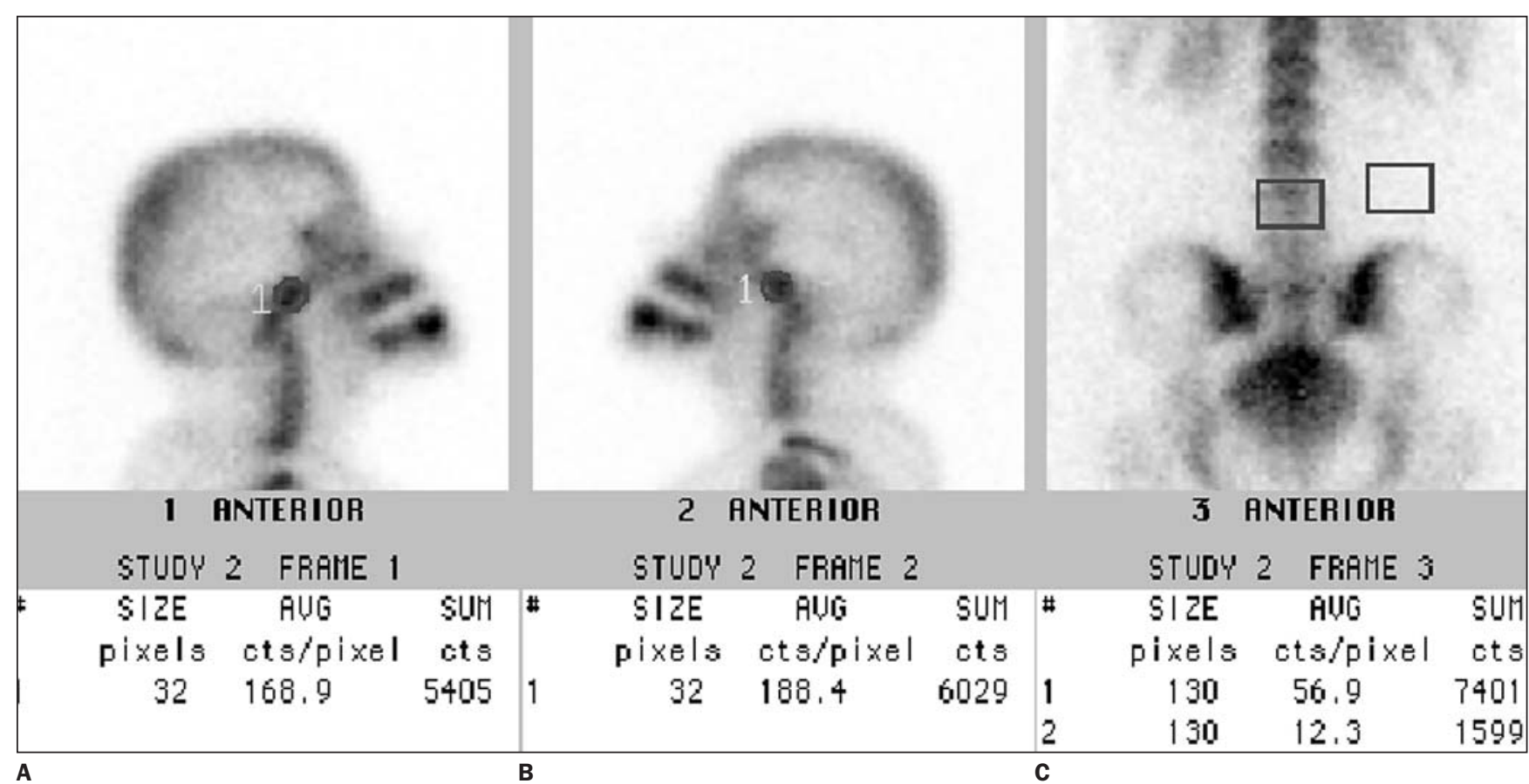

Figura 1. A,B: Cintilografia óssea de face, imagens lateral do côndilo direito (A) e lateral do côndilo esquerdo (B). C: Imagem da coluna lombo-sacra.

contagem do côndilo mandibular, da quarta vértebra lombar e do BKG, como no cálculo que segue:

Taxa de contagem = contagem na área de interesse na mandíbula - BKG / contagem na quarta vértebra lombar - BKG

O teste $t$ de Student foi utilizado para avaliar a freqüência das variáveis estudadas. A partir desses testes, p-valores inferiores a 0,05 foram considerados como estatisticamente significativos para as variáveis envolvidas.

\section{RESULTADOS}

A distribuição dos pacientes com crescimento facial equilibrado - classe I, grupo controle - mostrou maior freqüência de indivíduos do sexo masculino, em $69 \%$ (9/13) dos casos. Apesar da faixa etária proposta ter variado dos 18 aos 28 anos, apenas indivíduos com 18 e 19 anos realizaram o exame, havendo predomínio na idade de 18 anos (Tabela 1).

$\mathrm{Na}$ distribuição da amostra quanto ao sexo e a idade do grupo com deformidade esquelética de classe III — grupo casohouve discreto predomínio do sexo feminino, com 56\% (19/34) dos casos. A faixa etária variou dos 18 aos 24 anos, com

Tabela 1 Média de captação condilar do ${ }^{99 m}$ Tc-MDP do grupo controle através da contagem.

\begin{tabular}{|c|c|c|c|c|}
\hline \multirow[b]{2}{*}{ Paciente } & \multirow[b]{2}{*}{ Idade (anos) } & \multirow[b]{2}{*}{ Sexo } & \multicolumn{2}{|c|}{$\begin{array}{l}\text { Método contagem (300.000 contagens) } \\
\text { Região condilar/quarta vértebra lombar }\end{array}$} \\
\hline & & & Lado direito & Lado esquerdo \\
\hline 1 & 18 & Masculino & 3,01 & 2,95 \\
\hline 2 & 19 & Feminino & 2,22 & 1,11 \\
\hline 3 & 18 & Masculino & 1,96 & 2,06 \\
\hline 4 & 19 & Feminino & 3,70 & 3,90 \\
\hline 5 & 19 & Masculino & 1,66 & 1,63 \\
\hline 6 & 18 & Masculino & 2,83 & 2,64 \\
\hline 7 & 18 & Masculino & 3,90 & 3,90 \\
\hline 8 & 19 & Masculino & 2,54 & 2,52 \\
\hline 9 & 18 & Masculino & 2,78 & 2,95 \\
\hline 10 & 18 & Masculino & 2,39 & 2,20 \\
\hline 11 & 19 & Feminino & 2,05 & 2,29 \\
\hline 12 & 18 & Masculino & 2,12 & 2,44 \\
\hline 13 & 18 & Feminino & 4,06 & 3,65 \\
\hline
\end{tabular}

média de 20,4 anos para homens e 21,4 anos para as mulheres (Tabela 2).

Quando foram comparadas as médias de captação condilar dos lados direito e esquerdo para cada grupo, aplicando o teste $t$ de Student pareado, obtivemos $\mathrm{p}$-valor $=$ 0,427 para o grupo caso e $\mathrm{p}$-valor $=0,477$ para o grupo controle, o que indicou ausência de captação assimétrica condilar. A diferença entre os lados variou de $4 \%$ a $7 \%$. A simetria dos resultados permitiu agrupar os resultados dos lados esquerdo e direito (Tabela 3).

Quando foram comparadas as médias de captação condilar em relação à quarta vértebra lombar, nas projeções cintilográficas, não foram encontradas diferenças estatisticamente significativas ( $\mathrm{p}$-valor = 0,27 ) entre a atividade de crescimento entre os grupos caso e controle, de acordo com aplicação do teste $t$ de Student. Embora não tenhamos encontrado diferença 
Tabela 2 Média de captação condilar do ${ }^{99 m}$ Tc-MDP do grupo caso através da contagem.

\begin{tabular}{|c|c|c|c|c|}
\hline \multirow[b]{2}{*}{ Paciente } & \multirow[b]{2}{*}{ Idade (anos) } & \multirow[b]{2}{*}{ Sexo } & \multicolumn{2}{|c|}{$\begin{array}{l}\text { Método contagem ( } 300.000 \text { contagens) } \\
\text { Região condilar/quarta vértebra lombar }\end{array}$} \\
\hline & & & Lado direito & Lado esquerdo \\
\hline 1 & 21 & Masculino & 3,62 & 2,84 \\
\hline 2 & 20 & Masculino & 2,52 & 2,69 \\
\hline 3 & 23 & Feminino & 2,03 & 1,93 \\
\hline 4 & 19 & Feminino & 2,39 & 2,02 \\
\hline 5 & 18 & Masculino & 2,63 & 2,21 \\
\hline 6 & 18 & Masculino & 0,83 & 0,67 \\
\hline 7 & 24 & Feminino & 1,06 & 0,83 \\
\hline 8 & 19 & Feminino & 2,86 & 3,21 \\
\hline 9 & 24 & Masculino & 1,56 & 1,60 \\
\hline 10 & 24 & Feminino & 3,37 & 3,81 \\
\hline 11 & 27 & Feminino & 4,48 & 3,68 \\
\hline 12 & 28 & Feminino & 3,28 & 3,29 \\
\hline 13 & 18 & Feminino & 3,51 & 3,46 \\
\hline 14 & 18 & Feminino & 2,55 & 2,56 \\
\hline 15 & 18 & Feminino & 2,92 & 2,97 \\
\hline 16 & 23 & Masculino & 3,31 & 3,33 \\
\hline 17 & 18 & Feminino & 2,48 & 3,03 \\
\hline 18 & 18 & Feminino & 2,67 & 2,55 \\
\hline 19 & 23 & Feminino & 3,21 & 3,33 \\
\hline 20 & 24 & Feminino & 3,22 & 3,28 \\
\hline 21 & 18 & Masculino & 2,42 & 2,37 \\
\hline 22 & 18 & Feminino & 3,93 & 3,87 \\
\hline 23 & 20 & Feminino & 2,43 & 2,68 \\
\hline 24 & 27 & Feminino & 2,04 & 2,02 \\
\hline 25 & 20 & Masculino & 3,01 & 3,03 \\
\hline 26 & 18 & Masculino & 2,27 & 1,93 \\
\hline 27 & 19 & Masculino & 2,67 & 2,44 \\
\hline 28 & 22 & Masculino & 2,61 & 2,61 \\
\hline 29 & 20 & Feminino & 2,46 & 2,29 \\
\hline 30 & 18 & Masculino & 2,44 & 2,54 \\
\hline 31 & 19 & Masculino & 3,21 & 3,83 \\
\hline 32 & 22 & Feminino & 2,90 & 2,83 \\
\hline 33 & 28 & Masculino & 2,91 & 2,81 \\
\hline 34 & 20 & Masculino & 2,58 & 2,43 \\
\hline
\end{tabular}

significativa, a média de captação do grupo caso $(2,71)$ foi discretamente maior, em comparação à média encontrada no grupo controle $(2,63)$ (Gráfico 1).

\section{DISCUSSÃO}

Desde os primórdios, a ortodontia demonstra interesse tanto no estudo da movimentação dentária (efeito ortodôntico) quanto na possibilidade de alteração do crescimento e na posição das bases ósseas apicais (efeito ortopédico). O primeiro tema acha-se bem caracterizado, devido à facilidade de observação causa-efeito, mas o segundo ainda é controverso. Parte dos pesquisadores, apoiados na teoria da matriz funcional, acredita que os fatores ambientais locais sejam os principais responsáveis pelo tamanho final do esqueleto e, conseqüentemente, passível de certa regulação. A outra corrente advoga que o controle preponderante é genético, não afetando o crescimento das bases ósseas ${ }^{(22)}$.

A utilização da cintilografia óssea tem como vantagens a grande sensibilidade, a baixa agressividade, a alta especificidade do órgão e a baixa radiação ${ }^{(\mathbf{1 , 2 , 2 3 - 2 5 )}}$. A
Tabela 3 Análise da simetria entre côndilos direito e esquerdo dos grupos caso e controle.

\begin{tabular}{|l|c|c|}
\hline & Grupo caso & Grupo controle \\
\hline Média & 0,04 & 0,07 \\
Desvio-padrão & 0,3 & 0,3 \\
p-valor & 0,427 & 0,477 \\
\hline
\end{tabular}

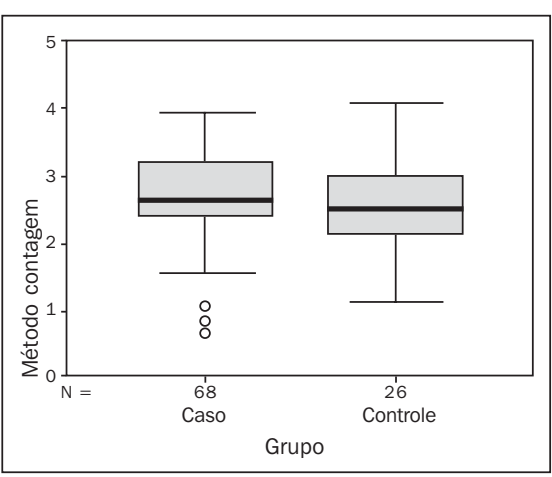

Gráfico 1. Box-plot da razão de captação condilar dos grupos caso e controle, agrupando os côndilos direito e esquerdo como um todo para cada grupo.

quantidade de radiação emitida durante o exame é comparável à utilizada para a radiografia de corpo inteiro numa criança, ou uma série padrão de radiografias cranianas em adultos ${ }^{(\mathbf{1})}$. A quantidade de radiação é determinada pela quantidade de radiofármaco utilizado, independente do número de imagens obtidas ${ }^{(\mathbf{1})}$. Por esses motivos, e por se tratar de especialidade médica raramente utilizada pela odontologia, optamos por estudar o uso da cintilografia óssea na verificação da atividade condilar em pacientes com prognatismo mandibular.

A cintilografia óssea mostrou-se um método promissor na verificação da atividade condilar e na dinâmica do crescimento facial, tendo consistência para a escolha do tratamento apropriado, do momento ideal para a realização de cirurgias, como também no acompanhamento das terapias ortodônticas e cirúrgicas ${ }^{(\mathbf{1}, 2,10,15,18,26,27)}$. Podemos observar, neste estudo, a facilidade de aplicação do método e de interpretação dos resultados, observando sempre os critérios de padronização na posição da cabeça, na identificação das áreas de interesse e no processamento das imagens.

Quando comparadas, as médias de captação condilar dos lados direito e esquerdo para cada grupo, aplicando o teste $t$ de Student pareado, obtivemos resultados que 
indicaram não haver diferença entre os lados, ou seja, ausência de captação condilar assimétrica. Encontramos resultados homogêneos, que variaram de $4 \%$ a $7 \%$. Cisneros e Kaban ${ }^{(\mathbf{1})}$ e Hodder et al. ${ }^{(\mathbf{1 0})}$ consideraram diferenças intercondilares em até $10 \%$ como simétricas, enquanto Bohuslavizki et al. ${ }^{(28)}$, que variações de $5 \%$ a $10 \%$ sejam casos limítrofes, merecendo acompanhamento periódico.

As médias da captação condilar dos pacientes prognatas encontradas neste trabalho foram diferentes daquelas descritas por Kaban et al. ${ }^{(\mathbf{2 , 3})}$. Duas hipóteses foram cogitadas: uma atividade condilar bastante aumentada, indicando um real potencial de crescimento mandibular, ou diferentes resultados causados pela seleção da amostra. Os autores mencionados utilizaram 90 indivíduos entre 2 e 30 anos de idade, porém não revelaram a distribuição quanto à faixa etária nem classificaram a amostra quanto ao padrão esquelético facial, como realizado no presente estudo. Houve então a necessidade da definição de um grupo controle específico para o padrão facial. As médias da captação do grupo controle também foram maiores que aquelas descritas nos trabalhos de Kaban et al. ${ }^{(\mathbf{2 , 3})}$ (Tabela 4). Embora não tenhamos encontrado diferença significativa, a média de captação do grupo caso foi discretamente maior, em comparação à média encontrada no grupo controle, sugerindo maior atividade metabólica condilar.

O tratamento de pacientes em crescimento, com deformidade mandibular, representa um problema tanto para o ortodontista como para o cirurgião. Os procedimentos cirúrgicos necessários para a correção da deformidade parecem não afetar o crescimento mandibular pós-operatório e o desenvolvimento dentofacial. Caso o crescimento facial continue após o ato cirúrgico, os benefícios esperados da cirurgia terão resultados aquém do ideal. A mandíbula parece apresentar um potencial de crescimento residual, por toda a vida, que ainda não está bem esclarecido.

A predição do crescimento facial ainda não é totalmente possível. É particularmente difícil em pacientes adolescentes
Tabela 4 Média de captação do ${ }^{99 m}$ Tc-MDP de acordo com a faixa etária.

\begin{tabular}{|c|c|}
\hline Idade & Média de captação \\
\hline $0-2$ & $2,00-1,85$ \\
$2-5$ & $1,85-1,65$ \\
$5-10$ & $1,65-1,30$ \\
$10-15$ & $1,30-1,10$ \\
$15-20$ & $1,10-0,70$ \\
$>20$ & $<0,70$ \\
\hline
\end{tabular}

com má-oclusão esquelética de classe III $^{(29)}$. Aqueles com verdadeiro prognatismo mandibular demonstram crescimento desproporcional sagital e vertical que continua durante alguns anos após a puberdade. Técnicas como avaliação de altura e peso, história de crescimento recente, estágios de ossificação dos ossos do punho e da mão são notoriamente imprecisos. A tomografia e a ressonância da articulação temporomandibular, embora permitam uma visão clara do tamanho e formato dos côndilos, fornecem poucas evidências sobre o crescimento futuro ${ }^{(\mathbf{1 4})}$.

São necessários mais estudos, ampliando a amostragem e estratificando-a de acordo com a idade cronológica ou biológica, para determinar valores padrões de atividade condilar e por meio deles identificar potencial de crescimento mandibular.

\section{REFERÊNCIAS}

1. Cisneros GJ, Kaban LB. Computerized skeletal scintigraphy for assessment of mandibular asymmetry. J Oral Maxillofac Surg 1984;42:513-20.

2. Kaban LB, Cisneros GJ, Heyman S, Treves S. Assessment of mandibular growth by skeletal scintigraphy. J Oral Maxillofac Surg 1982;40:18-22.

3. Kaban LB, Treves ST, Hattner RS, Pogrel MA. Skeletal scintigraphy for assessment of mandibular growth and asymmetry. In: Treves ST, ed. Pediatric nuclear medicine. 2nd ed. New York: Springer Verlag, 1995:316-27.

4. Casati AL, Tavano O. Curso de radiologia odontológica. 5aㅡ ed. São Paulo: Santos, 2000:248.

5. Freitas A, Rosa JE, Souza IF. Radiologia odontológica. 5a a ed. São Paulo: Artes Médicas, 2000: 583-90.

6. Siqueira VCV, Martins DR, Canuto CE, Janson GRP. O emprego das radiografias da mão e do punho do diagnóstico ortodôntico. Dental Press 1999;4:20-9.

7. Moore RN, Moyer BA, DuBois LM. Skeletal maturation and craniofacial growth. Am J Orthod Dentofacial Orthop 1990;98:33-40.

8. Moyers RE, Enlow DH. Crescimento do esquele- to craniofacial. In: Moyers RE. Ortodontia. 4⿳亠丷厂 ed. Rio de Janeiro: Guanabara Koogan, 1991:33-58.

9. Proffit WR. Ortodontia contemporânea. $3^{\mathrm{a}}$ ed. Rio de Janeiro: Guanabara Koogan, 2002.

10. Hodder SC, Rees JI, Oliver TB, Facey PE, Sugar AW. SPECT bone scintigraphy in the diagnosis and management of mandibular condylar hyperplasia. Br J Oral Maxillofac Surg 2000;38:87-93.

11. Enlow DH. Crescimento facial. $3^{3}$ ed. São Paulo: Artes Médicas, 1993.

12. Ferreira FV. Ortodontia: diagnóstico e planejamento clínico. 5ª ed. São Paulo: Artes Médicas, 2002: 187-223.

13. Kruger GO. Cirurgia bucal e maxilo-facial. 5e ed Rio de Janeiro: Guanabara Koogan, 1984.

14. Proffit WR, White RP. Surgical-orthodontic treatment. St. Louis: Mosby Year Book, 1991.

15. Epker BN, Stella JP, Fish LC. Dentofacial deformities: integrated orthodontic and surgical correction. 2nd ed. St. Louis: Mosby, 1994:611-7.

16. Medeiros PJ, Medeiros PP. Cirurgia ortognática para o ortodontista. São Paulo: Santos, 2001:29_ 56, 241-8.

17. Mojdehi M, Buschang PH, English JD, Wolford LM. Postsurgical growth changes in the mandible of adolescents with vertical maxillary excess growth pattern. Am J Orthod Dentofacial Orthop 2001;119:106-16.

18. Paulsen HU, Rabol A, Sorensen SS. Bone scintigraphy of human temporomandibular joints during Herbst treatment: a case report. Eur J Orthod 1998; 20:369-74

19. Morelli CG, Davidowicz H, Moura AAM. Cintilografia x ressonância magnética: revisão da literatura. Rev Inst Ciênc Saúde 1996;14:81-5.

20. Saha GB. Fundamentals of nuclear pharmacy. New York: Springer-Verlag, 1984.

21. Araújo TM. Cefalometria - conceitos e análises. (Dissertação de Mestrado). Rio de Janeiro: FOUFRJ, 1983.

22. Rabie ABM, Hagg U. Factors regulating mandibular condylar growth. Am J Orthod Dentofacial Orthop 2002;122:401-9.

23. Murray IPC. Bone scintigraphy: the procedure and interpretation. In: Murray IPC, Ell RJ, eds. Nuclear medicine in clinical diagnosis and treatment. Edinburgh: Churchill Livingstone, 1994.

24. Thrall JH, Ziessman HA. Medicina nuclear. $2^{\underline{a}}$ ed. Rio de Janeiro: Guanabara Koogan, 2003.

25. Piqueras JL, Tejedor JPT, Ariz IS, Martinez-Aedo JL, Mayorga AS. Medicina nuclear clínica. Madrid: Marban Editora, 1994.

26. Henderson MJ, Wastie ML, Bromige M, Selwyn P Smith A. Technetium-99m bone scintigraphy and mandibular condylar hyperplasia. Clin Radiol 1990;41:411-4.

27. Robinson PD, Harris K, Coghlan KC, Altman K. Bone scans and the timing of treatment for condylar hyperplasia. Int J Oral Maxillofac Surg 1990; 19:243-6.

28. Bohuslavizki KH, Brenner W, Kerscher A, et al. The value of bone scanning in pre-operative decisionmaking in patients with progressive facial asymmetry. Nucl Med Commun 1996;17:562-7.

29. Capelozza FL, Silva FOG. Crescimento craniofacial pós-natal. Apostila do Hospital de Pesquisa e Reabilitação de Lesões Lábio-Palatais. Bauru: USP/Bauru, 1995. 